\section{Meta-analyses with rare events should use adequate methods To the Editor:}

With great interest we read the meta-analysis of randomized clinical trials of Takagi and associates $^{1}$ on postoperative stroke risk in off-pump and on-pump coronary artery bypass grafting. Their work is a welcome update of the most recent metaanalysis on this topic by Sedrakyan and colleagues. $^{2}$

Contrary to Sedrakyan and associates, however, Tagaki and colleagues do not find a significant difference between offand on-pump surgery (relative risk [95\% confidence interval]: 0.60 [0.34-1.06]; $P=.08$ ), whereas Sedrakyan's group found 0.50 [0.27,0.93; $P=.03]$ for the relative risk. As an explanation, Tagaki's group points to the methodical difficulties of dealing with studies reporting no events in both treatment groups. Whereas Sedrakyan and associates removed these studies from analysis, Tagaki and colleagues obviously used a " 0.5 -correction" rule, where 0.5 is added to the number of events as well as to the number of nonevents in both groups (at least, this is how we were able to reproduce their results).

However, we object to both ideas of dealing with studies with zero events. Inasmuch as those studies point to equal risk for both treatments (as Tagaki's group noted correctly), deleting them, as Sedrakyan's group did, would probably overestimate the treatment effect. On the contrary, using the "0.5-correction" rule, as Tagaki and colleagues did, adds 13 pseudoevents in the off-pump group and another 13 in the on-pump group. Thus, the analysis is performed with 59 pseudo-observed, instead of 33 actually observed, events, rendering the analysis also somewhat dubious.

We would rather recommend methods that adequately account for studies with zero observations. These methods have been proposed, ${ }^{3}$ are straightforward extensions of the familiar Mantel-Haenszel method, ${ }^{4}$ and standard software (eg, SAS PROC FREQ, CMH option, code is available from the authors on request) is available for computation. We reanalyzed the Tagaki data* and found an estimated relative risk of 0.376 [0.175, 0.807; $P=.0091]$. Note that this value compares very closely with the relative risk from the simple and most intuitive analysis performed by collapsing the data from the 32 studies into a simple 4-fold table: 0.377 [0.176, 0.810; $P=.0092$ ].

We therefore conclude that there is still significant evidence that the off-pump method is superior to the on-pump method in reducing postoperative stroke risk after coronary bypass grafting.

Oliver Kuss, Dr sc hum Institute of Medical Epidemiology, Biostatistics, and Informatics

Medical Faculty, University of Halle-Wittenberg Halle (Saale), Germany Jan F. Gummert, Prof Dr med

Jochen Börgermann, Dr med Department of Cardiac and Thoracic Surgery Medical Faculty

Friedrich Schiller University Jena Jena, Germany

[Response declined]

* Note a printing error in Table 1 of the study by Tagaki and associates. ${ }^{1}$ The number of randomized patients in the off-pump group from the Lonn study should be " 15 ," not " 60 ." We performed all analyses with the corrected data set.

\section{References}

1. Takagi H, Tanabashi T, Kawai N, Umemoto T. Off-pump surgery does not reduce stroke, compared with results of on-pump coronary artery bypass grafting: a meta-analysis of randomized clinical trials. $J$ Thorac Cardiovasc Surg. 2007;134:1059-60

2. Sedrakyan A, Wu AW, Parashar A, Bass EB, Treasure T. Off-pump surgery is associated with reduced occurrence of stroke and other morbidity as compared with traditional coronary artery bypass grafting: a meta-analysis of systematically reviewed trials. Stroke. 2006;37:2759-69.

3. Greenland S, Robins JM. Estimation of common effect parameter from sparse follow-up data. Biometrics. 1985;41:55-68.

4. Mantel N, Haenszel W. Statistical aspects of the analysis of data from retrospective studies of disease. J Natl Cancer Inst. 1959;22:719-48.

doi:10.1016/j.jtcvs.2007.12.046

\section{Factors predicting poor survival after resection of stage IA non-small cell lung cancer To the Editor:}

We have read with interest the paper by Chang and associates. ${ }^{1}$ Interestingly, we observe that the factors considered to predict poor survival after resection of stage IA non-small cell lung cancer (NSCLC) still remain tumor size, gender, age, and extent of resection.
It is true, in fact, that lung cancer staging currently rests on histopathologic and clinical criteria that have only limited power to predict relapse and survival. A major effort to improve the control of NSCLC entails the use of molecular profiling to characterize tumors and provide accurate predictions of the outcome after standard or novel treatments. Moreover, molecular profiling, as $w^{2}$ already discussed in 2003 , could really provide an entirely new classification system.

Recently, one study has demonstrated the potential clinical applications of gene expression profiling in a cohort of 89 patients with early-stage NSCLC in predicting the risk of disease recurrence. ${ }^{3}$ The authors evaluated the predictor in two independent groups of 25 patients from the American College of Surgeons Oncology Group Z0030 study and 84 patients from the Cancer and Leukemia Group B 9761 study. The overall predictive accuracy was $72 \%$ and $79 \%$, respectively. The predictor also identified a subgroup of patients with stage IA disease who were at high risk for recurrence and who might be best treated by adjuvant chemotherapy. Additionally, an 11-gene expression signature associated with "stem cellness" was found to divide patients with different cancers, including NSCLC, into good- and poor-prognosis groups; however, this stem cell-associated signature has not been validated or further studied in NSCLC. ${ }^{4}$ On a pragmatic basis, a rigorous prospective approach, using training and testing cohorts, to study molecular prognostic markers could improve chances of identifying true molecular prognostic markers that may be reliably applied to clinical practice. Potential research goals may include the following (1): identify molecular tissue, blood, and plasma markers (ie, gene expression profile, genetic polymorphism, genetic/epigenetic alterations, plasma proteomic) predictive of survival, recurrence, and metastasis development in patients with NSCLC; (2) establish characteristics of precursor lesions and the field of cancerization phenomenon in NSCLC pathogenesis by smoking status, gender, and ethnic background; (3) establish molecular markers to discover occult micrometastasis in lymph nodes (sentinel lymph node); (4) evaluate the presence of "stem-cancer cells"; (5) identify molecular tissue and blood markers to predict response to adjuvant chemotherapy; (6) identify molecular markers predictive of response to chemotherapeutic 


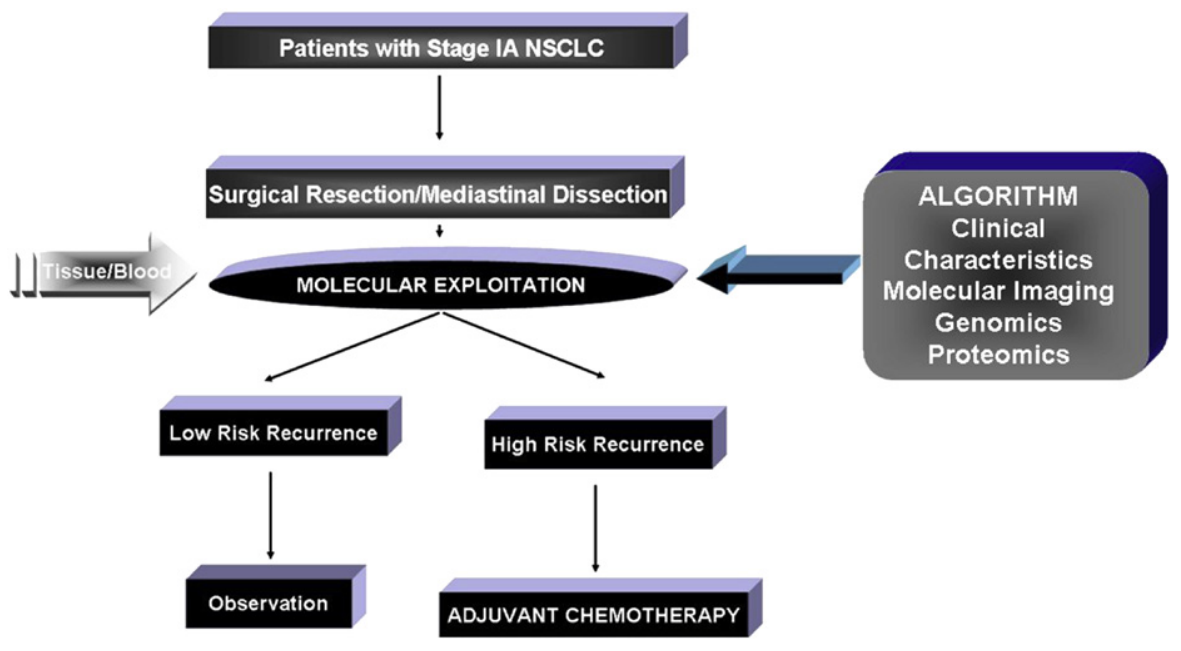

Figure 1. Application of molecular biology to refine the assessment of risk and guide the use of adjuvant chemotherapy in stage IA non-small cell lung cancer (NSCLC).

or targeted therapeutic agents at time of recurrence. Therefore, development of a tissue and blood (serum, plasma, and circulating cells) bank with specimens obtained in clinical trials, including detailed prospective collected clinical data, is of the utmost importance. This new phase of target profiling and agent-specific profiling will probably require an algorithm that would include genomic, proteomic, clinical, and imaging factors.

Patients with early-stage NSCLC will be assigned to particular drugs on the basis of the molecular characteristics of their tumors. Then the development of drugs for the treatment of NSCLC will be focused on personalized therapy.

Figure 1 outlines a possible application of molecular biology to refine the assessment of risk and guide the use of adjuvant chemotherapy in stage IA NSCLC.

Laura Paleari, $P h D^{a}$

Patrizia Russo, $P h D^{a}$ Alfredo Cesario, $M D, P h D^{b, c}$ Pierluigi Granone, $M D, P h D^{b}$

Lung Cancer Unit National Cancer Research Institute Genoa, Italy ${ }^{a}$

Surgical Pathology Unit Catholic University Rome, Italy

Respiratory Unit IRCCS "San Raffaele" Rome, Italy"

\section{References}

1. Chang MY, Mentzer SJ, Colson YL, Linden PA, Jaklitsch MT, Lipsitz SR, et al. Factors predicting poor survival after resection of stage IA non-small cell lung cancer. $J$ Thorac Cardiovasc Surg. 2007;134:850-6.

2. Cesario A, Galletta D, Russo P, Margaritora S, Granone P. The role of the surgeon in translational research. Lancet. 2003;362: 1082.

3. Potti A, Mukherjee S, Petersen R, Dressman HK, Bild A, Koontz J, et al. A genomic strategy to refine prognosis in early-stage non-small-cell lung cancer. N Engl J Med. 2006;355:570-80.

4. Glinsky GV, Berezovska O, Glinskii AB. Microarray analysis identifies a death-from-cancer signature predicting therapy failure in patients with multiple types of cancer. $J$ Clin Invest. 2005;115:1503-21.

\section{doi:10.1016/j.jtcvs.2007.11.056}

\section{Focus on the Thoracoscore \\ To the Editor:}

It is with great interest that we read the article entitled "Thoracoscore predicts midterm mortality in patients undergoing thoracic surgery." The authors' findings and conclusions concur perfectly with those of our initial main work, which first created and proposed the use of the Thoracoscore. ${ }^{2}$ However, we would like to emphasize 2 points that we consider to be important.

First, one of our concerns is the reliability of any modification in the French scoring system (Thoracoscore), as was done with the dyspnea score in the present study. This point is particularly crucial. Effectively, our initial aim was to develop a self-assessment system based on a large database reflecting current practices in adult thoracic surgery. Nine predictors were selected from the database. Their definitions, which are consistent throughout the world and particularly in North American systems, were objective enough to develop a reliable scoring system that could be used by all thoracic surgeons and for all types of thoracic procedures. This is why we think that the Thoracoscore can be used as a relevant yardstick for mortality. Moreover, the French scoring system, built on well-defined risk factors, is also user friendly and imposes minimal demands on medical time. At this point, a word of caution is essential: the condition under which such a system is used needs to be considered. The Thoracoscore was constructed so as to deal with the problem of a missing item. Indeed, in the case of 1 or more items missing from the 9 predictors selected, the Thoracoscore gives a range (minimum and maximum values) of estimated mortality rather than a percentage of predictive mortality. Thus it can be used easily, as is.

Second, why is the Thoracoscore a reliable tool, and how can it be used worldwide? The Thoracoscore is reliable because it comes from a national electronic prospective database created by a national society of thoracic and cardiovascular surgery. Indeed, the French database Epithor, created in 2002 on behalf of the French Society of Thoracic and Cardiovascular Surgery, includes anonymous information from two thirds of all thoracic surgery procedures performed in France each year (>60,000 from 59 centers: 37 public and 15 private). Each center is free to compare its statistics and results with the national means. It can also be used to register patients for administrative hospital files. In addition, Epithor is now mandatory for the accreditation of thoracic surgery departments. Therefore the Thoracoscore tool can be transposed to any organization (country/ 